\title{
On the Optimality of Conditional Expectation as a Bregman Predictor
}

\author{
Arindam Banerjee \\ Department of Electrical and Computer Engineering \\ University of Texas at Austin \\ Austin, TX 78712. \\ abanerje@ece.utexas.edu \\ Xin Guo \\ School of Operations Research and Industrial Engineering \\ Cornell University \\ Ithaca, NY 14583. \\ xinguo@orie.cornell.edu \\ Hui Wang \\ Division of Applied Mathematics \\ Brown University \\ Providence, RI 02912. \\ huiwang@cfm.brown.edu
}

August 11, 2003

\begin{abstract}
Given a probability space $(\Omega, \mathcal{F}, P)$, a $\mathcal{F}$-measurable random variable $X$, and a sub- $\sigma$-algebra $\mathcal{G} \subset \mathcal{F}$, it is well known that the conditional expectation $E[X \mid \mathcal{G}]$ is the optimal $\mathbb{L}^{2}$-predictor (also known as "the least mean square error" predictor) of $X$ among all the $\mathcal{G}$-measurable random variables $[8,11]$. In this paper, we provide necessary and sufficient conditions under which the conditional expectation is the unique optimal predictor. We show that $E[X \mid \mathcal{G}]$ is the optimal predictor for all Bregman Loss Functions (BLFs), of which $\mathbb{L}^{2}$ loss function is a special case. Moreover, under mild conditions, we show that BLFs are exhaustive. Namely, if the infimum of $E[F(X, Y)]$ over all the $\mathcal{G}$-measurable random variables $Y$ and for any variable $X$ is attained at the conditional expectation $E[X \mid \mathcal{G}]$, then $F$ is a BLF.
\end{abstract}




\section{Introduction}

It arises in many contexts that one would like to predict the value of a random outcome based on the available information. To put the problem into a mathematical framework, let $(\Omega, \mathcal{F}, P)$ be a probability space and $X$ an $\mathcal{F}$-measurable random variable that one wishes to predict. The available information is represented by a sub- $\sigma$-algebra of $\mathcal{F}$, say $\mathcal{G}$. Now the question is, among all the $\mathcal{G}$-measurable random variables, which one is the best predictor for $X$.

The notion of "best" is usually specified by a non-negative loss function $F$ and achieved by solving a corresponding minimization problem. More precisely, the best predictor is defined as the minimizer of $E[F(X, Y)]$ over all the $\mathcal{G}$-measurable random variables $Y$. A particularly important case is when $F$ is the so called the $\mathbb{L}^{2}$-loss function, also known as the mean square error; namely $F(x, y) \doteq\|x-y\|^{2}$. It is well known $[8,11]$ that the corresponding unique best predictor is given by the conditional expectation. In other words, if we write $Y \in \mathcal{G}$ for a $\mathcal{G}$-measurable random variable $Y$, then

$$
\underset{Y \in \mathcal{G}}{\operatorname{argmin}} E\left[\|X-Y\|^{2}\right]=E[X \mid \mathcal{G}] .
$$

This makes the conditional expectation a crucially important concept for prediction.

A question arises naturally: Are there other loss functions $F$ for which $E[X \mid \mathcal{G}]$ is the unique best predictor? Some simple counter-examples lead to the general conviction that the existence of such loss functions would be rare and would have to possess very special properties. For example [9] if one uses the absolute error loss function and take $\mathcal{G}=\{\emptyset, \Omega\}$, then any constant $a$ satisfying $P(X \leq a) \geq 1 / 2 \geq P(X>a)$, i.e., the median of $X$ and not $E[X \mid \mathcal{G}]$, proves to be the best predictor. Recently [1] studied the case of general convex loss functions and obtained a criterion for which a minimizing value exists when $\mathcal{G}=\mathcal{F}$.

In this paper, we provide necessary and sufficient conditions under which the conditional expectation is the unique optimal predictor. First, we show that the optimality property of the conditional expectation holds for all functions known as Bregman Loss Functions (BLFs) [3], of which the $\mathbb{L}^{2}$-loss function is a special case. Indeed, one can essentially create as many BLFs as convex functions, up to equivalences in linear and constant terms (see Definition 1). Secondly, we show that the class of BLFs is exhaustive under mild conditions. That is, if the conditional expectation minimizes the expected loss function for all random variables $X$, then the loss function has to be a BLF.

\section{Bregman Loss Functions}

Definition 1 (Bregman Loss Functions) Let $\phi: \mathbb{R}^{d} \mapsto \mathbb{R}$ be a strictly convex, differentiable function. Then, the Bregman Loss Function $D_{\phi}: \mathbb{R}^{d} \times \mathbb{R}^{d} \mapsto \mathbb{R}$ is defined as

$$
D_{\phi}(x, y)=\phi(x)-\phi(y)-\langle x-y, \nabla \phi(y)\rangle
$$


Table 1: Examples of BLFs.

\begin{tabular}{|l|c|c|c|}
\hline Domain & $\phi(x)$ & $D_{\phi}(x, y)$ & Loss \\
\hline $\mathbb{R}$ & $x^{2}$ & $(x-y)^{2}$ & $\mathbb{L}^{2}$-loss \\
\hline $\mathbb{R}_{++}$ & $x \log x$ & $x \log (x / y)-(x-y)$ & Logistic loss \\
\hline$(0,1)$ & $x \log x+(1-x) \log (1-x)$ & $x \log (x / y)+(1-x) \log ((1-x) /(1-y))$ & Itakura-Saito distance \\
\hline $\mathbb{R}_{++}$ & $-\log x$ & $x / y-\log (x / y)-1$ & $\mathbb{L}^{2}$-loss \\
\hline $\mathbb{R}$ & $e^{x}$ & $e^{x}-e^{y}-(x-y) e^{y}$ & Mahalanobis distance ${ }^{1}$ \\
\hline $\mathbb{R}^{d}$ & $\|x\|^{2}$ & $(x-y)^{T} A(x-y)$ & KL-divergence \\
\hline $\mathbb{R}^{d}$ & $x^{T} A x$ & $\sum_{j=1}^{d} x_{j} \log \left(x_{j} / y_{j}\right)$ & Generalized I-divergence \\
\hline$d$-simplex & $\sum_{j=1}^{d} x_{j} \log x_{j}$ & & \\
\hline $\mathbb{R}_{+}^{d}$ & $\sum_{j=1}^{d} x_{j} \log x_{j}$ & $\sum_{j=1}^{d} x_{j} \log \left(x_{j} / y_{j}\right)-\sum_{j=1}^{d}\left(x_{j}-y_{j}\right)$ & \\
\hline
\end{tabular}

Example 1: The well-known $\mathbb{L}^{2}$-loss function is the simplest and most widely used loss function. It is a special case of BLFs. Choosing $\phi(x) \doteq\langle x, x\rangle$, we have

$$
D_{\phi}(x, y)=\langle x, x\rangle-\langle y, y\rangle-\langle x-y, 2 y\rangle=\|x-y\|^{2} .
$$

Example 2: Another widely used BLF is the Kullback-Liebler divergence. Let $p \doteq$ $\left(p_{1}, \ldots, p_{d}\right)$ be a discrete probability distribution so that $\sum_{j=1}^{d} p_{j}=1$. The negative Shannon entropy, $\phi(p) \doteq \sum_{j=1}^{d} p_{j} \log p_{j}$, is a strictly convex function on the $d$-simplex. Let $q=\left(q_{1}, \ldots, q_{d}\right)$ be another probability distribution. The corresponding BLF is

$$
\begin{aligned}
D_{\phi}(p, q) & =\sum_{j=1}^{d} p_{j} \log p_{j}-\sum_{j=1}^{d} q_{j} \log q_{j}-\langle p-q, \nabla \phi(q)\rangle \\
& =\sum_{j=1}^{d} p_{j} \log p_{j}-\sum_{j=1}^{d} q_{j} \log q_{j}-\sum_{j=1}^{d}\left(p_{j}-q_{j}\right)\left(\log e+\log q_{j}\right) \\
& =\sum_{j=1}^{d} p_{j} \log \left(p_{j} / q_{j}\right),
\end{aligned}
$$

which is exactly the KL-divergence $K L(p \| q)$ between $p$ and $q$.

The following useful observation follows from the strictly convexity of $\phi[6$, Proposition $5.4]$.

Lemma 1 For any $x, y \in \mathbb{R}^{d}, D_{\phi}(x, y) \geq 0$, and the equality holds if and only if $x=y$.

Remark 1 Since a differentiable convex function is necessarily continuously differentiable [10, Theorem 25.5], the function $D_{\phi}$ is continuous. Moreover, if we write $\nabla_{x}$ as the gradient with respect to $x$, then the function

$$
\nabla_{x} D_{\phi}(x, y)=\nabla \phi(x)-\nabla \phi(y)
$$

\footnotetext{
${ }^{1}$ The matrix $A$ is assumed to be strictly positive definite.
} 
is also continuous. For more discussions on BLFs, interested readers are referred to [2] and the references therein.

\section{The optimal Bregman predictor}

In this section we will show that the conditional expectation is the unique optimal predictor for all BLFs, and that any nearly optimal predictor will converge in probability to the conditional expectation.

Theorem 1 (Optimality Property) Let $\phi: \mathbb{R}^{d} \mapsto \mathbb{R}$ be a strictly convex, differentiable function. Let $(\Omega, \mathcal{F}, P)$ be an arbitrary probability space and $\mathcal{G}$ a sub- $\sigma$-algebra of $\mathcal{F}$. Let $X$ be any $\mathcal{F}$-measurable random variable taking values in $\mathbb{R}^{d}$ for which both $E[X]$ and $E[\phi(X)]$ are finite. Then the conditional expectation is the unique minimizer (up to a.s. equivalence) for BLFs, i.e.,

$$
\operatorname{argmin}_{Y \in \mathcal{G}} E\left[D_{\phi}(X, Y)\right]=E[X \mid \mathcal{G}] .
$$

Proof: Let $Y$ be any $\mathcal{G}$-measurable random variable, and $Y^{*} \doteq E[X \mid \mathcal{G}]$. It follows from Definition 1 that

$$
\begin{aligned}
& E\left[D_{\phi}(X, Y)\right]-E\left[D_{\phi}\left(X, Y^{*}\right)\right] \\
& \quad=E\left[\phi\left(Y^{*}\right)-\phi(Y)-\langle X-Y, \nabla \phi(Y)\rangle+\left\langle X-Y^{*}, \nabla \phi\left(Y^{*}\right)\right\rangle\right] .
\end{aligned}
$$

Meanwhile, for any $\mathcal{G}$-measurable random variable $Y$, we have

$$
E[\langle X-Y, \nabla \phi(Y)\rangle]=E[E[\langle X-Y, \nabla \phi(Y)\rangle \mid \mathcal{G}]]=E\left[\left\langle Y^{*}-Y, \nabla \phi(Y)\right\rangle\right] .
$$

In particular, $E\left[\left\langle X-Y^{*}, \nabla \phi\left(Y^{*}\right)\right\rangle\right]=0$. Therefore,

$$
\begin{aligned}
E\left[D_{\phi}(X, Y)\right]-E\left[D_{\phi}\left(X, Y^{*}\right)\right] & =E\left[\phi\left(Y^{*}\right)-\phi(Y)-\left\langle Y^{*}-Y, \nabla \phi(Y)\right\rangle\right] \\
& =E\left[D_{\phi}\left(Y^{*}, Y\right)\right] .
\end{aligned}
$$

The Theorem follows immediately from Lemma 1.

Theorem 2 (Convergence in Probability) In the setting of Theorem 1, if $\left\{Y_{n}\right\}$ is an infimizing sequence, i.e., $Y_{n}$ is $\mathcal{G}$-measurable and

$$
E\left[D_{\phi}\left(X, Y_{n}\right)\right] \rightarrow E\left[D_{\phi}\left(X, Y^{*}\right)\right],
$$

where $Y^{*} \doteq E[X \mid \mathcal{G}]$, then $Y_{n} \rightarrow Y^{*}$ in probability.

Proof: It suffices to show that for any given $\epsilon, \delta>0$, there exists a number $N$ such that

$$
P\left(\left\|Y_{n}-Y^{*}\right\| \geq \delta\right) \leq \epsilon
$$


for all $n \geq N$. The integrability of $X$ (and hence of $Y^{*}$ ) suggests that there exists an $M$ such that

$$
P\left(\left\|Y^{*}\right\| \geq M\right) \leq \epsilon / 2 \text {. }
$$

Hence

$$
\begin{aligned}
P\left(\left\|Y_{n}-Y^{*}\right\| \geq \delta\right) & \leq P\left(\left\|Y_{n}-Y^{*}\right\| \geq \delta,\left\|Y^{*}\right\| \leq M\right)+P\left(\left\|Y^{*}\right\| \geq M\right) \\
& \leq P\left(\left\|Y_{n}-Y^{*}\right\| \geq \delta,\left\|Y^{*}\right\| \leq M\right)+\epsilon / 2 .
\end{aligned}
$$

Define for every $x \in \mathbb{R}^{d}$

$$
h(x) \doteq \inf \left\{D_{\phi}(x, y): y \in \mathbb{R}^{d},\|y-x\| \geq \delta\right\} .
$$

The strict convexity of $\phi$ implies that $h(x)>0$ for every $x \in \mathbb{R}^{d}$, and

$$
h(x)=\inf \left\{D_{\phi}(x, y): y \in \mathbb{R}^{d},\|y-x\|=\delta\right\} .
$$

Since $D_{\phi}$ is continuous (Remark 1), the infimum is always achieved. Moreover, it can be shown that

$$
\alpha \doteq \inf \{h(x):|x| \leq M\}>0 .
$$

For now assuming (2) is true, we have

$$
P\left(\left\|Y_{n}-Y^{*}\right\| \geq \delta\right) \leq P\left(D_{\phi}\left(Y^{*}, Y_{n}\right) \geq \alpha\right)+\epsilon / 2 \leq E\left[D_{\phi}\left(Y^{*}, Y_{n}\right)\right] / \alpha+\epsilon / 2 .
$$

Since $Y_{n}$ is an infimizing sequence, from (1) it follows that $E\left[D_{\phi}\left(Y^{*}, Y_{n}\right)\right] \rightarrow 0$. Hence, there exists $N$ such that for $n \geq N, E\left[D_{\phi}\left(Y, Y_{n}\right)\right] \leq \epsilon \alpha / 2$. Therefore, for $n \geq N$, we have

$$
P\left(\left\|Y_{n}-Y^{*}\right\| \geq \delta\right) \leq \epsilon,
$$

and hence convergence in probability.

Now, it remains to be shown that $\alpha>0$. This is proved by contradiction. Suppose $\alpha=0$. Then there exists a sequence $\left\{x_{n}\right\}$ with $\left\|x_{n}\right\| \leq M$ and a sequence $\left\{y_{n}\right\}$ with $\left\|y_{n}-x_{n}\right\|=\delta$ such that

$$
h\left(x_{n}\right)=D_{\phi}\left(x_{n}, y_{n}\right) \rightarrow 0 .
$$

Since $\left\{x_{n}\right\}$ and $\left\{y_{n}\right\}$ are both bounded, there exists a subsequence (still indexed by $n$ ) such that

$$
x_{n} \rightarrow \bar{x}, \quad y_{n} \rightarrow \bar{y} .
$$

Clearly $\|\bar{x}\| \leq M$ and $\|\bar{y}-\bar{x}\|=\delta$. The continuity of $D_{\phi}$ yields that $D_{\phi}(\bar{x}, \bar{y})=0$, which contradicts $h(\bar{x})>0$.

Now the proof is complete.

Remark 2 Stronger and different types of convergence results may be obtained by imposing proper conditions on the function $\phi$. For example, it is easy to see that $Y_{n} \rightarrow Y^{*}$ in $\mathbb{L}^{2}$ if the Hessian matrix of $\phi$ is uniformly positive definite over $\mathbb{R}^{d}$ (in the 1-dim case, it amounts to $\left.\inf _{x \in \mathbb{R}} \phi^{\prime \prime}(x)>0\right)$. 


\section{Exhaustiveness property of BLF}

In this section we establish the exhaustive results for the class of BLFs. More precisely, we show, under mild regularity conditions, that if $F: \mathbb{R}^{d} \times \mathbb{R}^{d} \mapsto \mathbb{R}$ is a non-negative loss function such that

$$
\operatorname{argmin}_{Y \in \mathcal{G}} E[F(X, Y)]=E[X \mid \mathcal{G}],
$$

for all random variable $X$, then $F$ has to be a BLF.

We will present the results for the one-dimensional case (Theorem 3) and the higherdimensional case (Theorem 4) separately, since the latter needs slightly stronger regularity conditions; see section 5 for more discussions.

For ease of exposition, without loss of generality, we will assume $F(x, x)=0$ for any $x$ in Theorem 3 and Theorem 4 . Indeed, if $F$ is a loss function satisfying (3), so is $\bar{F}(x, y) \doteq F(x, y)-F(x, x)$ with $\bar{F}(x, x) \equiv 0$.

Theorem $3(d=1)$ Let $F: \mathbb{R} \times \mathbb{R} \mapsto \mathbb{R}$ be a non-negative function such that $F(x, x)=$ $0, \forall x \in \mathbb{R}$. Assume that $F$ and $F_{x}$ are both continuous functions. If for all random variables $X, E[X \mid \mathcal{G}]$ is the unique minimizer for $E[F(X, Y)]$ over random variables $Y \in \mathcal{G}$, i.e.,

$$
\operatorname{argmin}_{Y \in \mathcal{G}} E[F(X, Y)]=E[X \mid \mathcal{G}],
$$

then $F(x, y)=D_{\phi}(x, y)$ for some strictly convex, differentiable function $\phi: \mathbb{R} \mapsto \mathbb{R}$.

Proof: The proof will be completed in three steps. First, we prove that $F=D_{\phi}$ for some convex, differentiable function $\phi$, under an additional assumption that $F_{y}$ is continuous; We then extend this result to the general case by a mollification argument; Finally, we show that $\phi$ is strictly convex.

Step 1: Assuming $F_{x}, F_{y}$ are both continuous. Fix arbitrarily $a, b \in \mathbb{R}$, and $p \in[0,1]$. Consider a random variable $X$ on some probability space $(\Omega, \mathcal{F}, P)$ such that $P(X=a)=p$ and $P(X=b)=q$ with $p+q=1$. Let $\mathcal{G}=\{\emptyset, \Omega\}$. Fix $Y=y$, then by assumption

$$
p F(a, y)+q F(b, y)=E[F(X, Y)] \geq E[F(X, E X)]=p F(a, p a+q b)+q F(b, p a+q b)
$$

for all $y \in R$. Moreover, if we consider the left-hand-side as a function of $y$, it equals the right-hand-side at $y=y^{*} \doteq E[X]=p a+q b$. Therefore, we must have

$$
p F_{y}\left(a, y^{*}\right)+q F_{y}\left(b, y^{*}\right)=0 .
$$

Substituting $p=\left(y^{*}-b\right) /(a-b)$ and rearranging terms yield

$$
F_{y}\left(a, y^{*}\right) /\left(y^{*}-a\right)=F_{y}\left(b, y^{*}\right) /\left(y^{*}-b\right) .
$$

Since $a, b$ and $p$ are arbitrary, the above equality implies that the function

$$
F_{y}(x, y) /(y-x)
$$


is independent of $x$. Thus one can write, for some function $H$,

$$
F_{y}(x, y)=(y-x) H(y)
$$

where $H$ is continuous.

Now define function $\phi$ by

$$
\phi(y) \doteq \int_{0}^{y} \int_{0}^{t} H(s) d s d t
$$

Then $\phi$ is differentiable with $\phi(0)=\phi^{\prime}(0)=0, \phi^{\prime \prime}(y)=H(y)$. Since $F(x, x)=0$, integration by parts for (5) leads to

$$
F(x, y)=\int_{x}^{y}(s-x) H(s) d s=\phi(x)-\phi(y)-\phi^{\prime}(y)(x-y) .
$$

Step 2: Now we show that there exists a convex function $\phi$ such that $F=D_{\phi}$ under the assumption of the theorem. Consider a sequence of mollifier, i.e., a sequence of functions $\left\{g_{n}\right\}$ defined on $\mathbb{R}$, which are non-negative, $\mathcal{C}^{\infty}$, with compact support such that

$$
\int_{\mathbb{R}} g_{n}(x) d x=1
$$

A classical example for such a sequence of mollifier is as follows: let

$$
g(x) \doteq \begin{cases}c \exp \left\{1 /\left(x^{2}-1\right)\right\} & \text { if }|x|<1 \\ 0 & \text { if }|x| \geq 1,\end{cases}
$$

where the constant $c$ is to chosen so that $\int_{\mathbb{R}} g(x) d x=1$, and define $g_{n}(x) \doteq n g(n x)$. The mollified version of $F$ is then given by

$$
F_{n}(x, y) \doteq \int_{\mathbb{R}} F(x-u, y-u) g_{n}(u) d u=\int_{\mathbb{R}} F(x-y+u, u) g_{n}(y-u) d u .
$$

It is standard to show that $[7$, Section 7.2$] F_{n}$ is continuously differentiable with respect to $x$ and $y$, and that

$$
\lim _{n \rightarrow \infty} F_{n}(x, y)=F(x, y)
$$

for every $x, y \in \mathbb{R}$.

Further, it is easy to see that $F_{n}$ has the same property as $F$, i.e., $E[X \mid \mathcal{G}]$ is the minimizer for the loss function $F_{n}$. Therefore, by the proof in Step 1, there exists a convex, differentiable function $\phi_{n}$ such that $\phi_{n}(0)=\phi_{n}^{\prime}(0)=0$ and

$$
F_{n}(x, y)=\phi_{n}(x)-\phi_{n}(y)-\phi_{n}^{\prime}(y)(x-y) .
$$


In particular, $F_{n}(x, 0)=\phi_{n}(x)$. Since $F_{n}(x, 0) \rightarrow F(x, 0)$ for every $x$, we have

$$
\lim _{n \rightarrow \infty} \phi_{n}(x)=F(x, 0) \doteq \phi(x)
$$

for every $x$. Since $\phi_{n}$ 's are convex, so is their limit $\phi$. In particular, $\phi$ is continuous $[10$, Theorem 10.1]. Setting $x=y+1$ in equation (6), we have

$$
\begin{aligned}
\phi_{n}^{\prime}(y) & =F_{n}(y+1, y)-\phi_{n}(y+1)+\phi_{n}(y) \\
\Rightarrow \lim _{n \rightarrow \infty} \phi_{n}^{\prime}(y) & =F(y+1, y)-\phi(y+1)+\phi(y) \doteq f(y) .
\end{aligned}
$$

Clearly $f$ is continuous. Letting $n \rightarrow \infty$ in both sides of equation (6), we have

$$
F(x, y)=\phi(x)-\phi(y)-f(y)(x-y) .
$$

Where $\phi$ is continuously differentiable, since $F$ is continuously differentiable with respect to $x$. Furthermore, the non-negativity of $F$ implies that $f(y)$ is a subgradient of $\phi[10$, Page 214]. Finally, the differentiability of $\phi$ suggests that its subdifferential is just its derivative [10, Theorem 25.1]. It follows that $\phi^{\prime}(y)=f(y)$, whence $F=D_{\phi}$.

Step 3: It remains to show that $\phi$ is strictly convex. From step 2, we already know that $\phi$ is a convex function. We argue by contradiction that if $\phi$ is not strictly convex, the assumption of uniqueness will be violated. Suppose $\phi$ is not strictly convex. Then there exists an interval $I=\left[\ell_{1}, \ell_{2}\right]$ such that $\ell_{1}<\ell_{2}$ and $\phi^{\prime}(y)=\phi^{\prime}\left(\ell_{1}\right)$ for all $y \in I$. Consider a random variable $X$ such that $P\left(X=\ell_{1}\right)=P\left(X=\ell_{2}\right)=1 / 2$, and set $\mathcal{G}=\{\emptyset, \Omega\}$. It is not difficult to check that any $y \in I$ is a minimizer. Indeed, $E\left[D_{\phi}(X, y)\right] \equiv 0$ for all $y \in I$. This is a contradiction and we complete the proof.

Theorem $4(d \geq 2)$ Let $F: \mathbb{R}^{d} \times \mathbb{R}^{d} \mapsto \mathbb{R}$ be a non-negative function such that $F(x, x)=$ $0, \forall x \in \mathbb{R}^{d}$. Assume that $F(x, y)$ and $F_{x_{i} x_{j}}(x, y), 1 \leq i, j \leq d$ are all continuous. If $E[X \mid \mathcal{G}]$ is the unique minimizer for $E[F(X, Y)]$ over all random variables $Y \in \mathcal{G}$ and for all random variables $X$ taking value in $\mathbb{R}^{d}$, i.e.,

$$
\operatorname{argmin}_{Y \in \mathcal{G}} E[F(X, Y)]=E[X \mid \mathcal{G}],
$$

then $F(x, y)=D_{\phi}(x, y)$ for some strictly convex and differentiable function $\phi: \mathbb{R}^{d} \mapsto \mathbb{R}$.

The proof is divided into three analogous steps as those in Theorem 3. The only essential difference is in Step 1, which relies on the following lemma. The lemma itself is a direct consequence of the celebrated Poincaré Lemma.

Lemma 2 Given a collection of continuous functions $\left\{h_{i j}: 1 \leq i, j \leq d\right\}$ defined on an open, convex set $U \subseteq \mathbb{R}^{d}$ (d $\geq 2$ ). If for all triples of indices $1 \leq i, j, k \leq d$,

$$
h_{i j} \equiv h_{j i}, \quad \frac{\partial h_{i j}}{\partial x_{k}} \equiv \frac{\partial h_{k j}}{\partial x_{i}} .
$$

Then there exists a function $\Phi: U \mapsto \mathbb{R}$ such that $\Phi_{x_{i} x_{j}}=h_{i j}$. 
Proof: (of Lemma 2) We first show that there exists a sequence of functions $\left\{\phi_{i}: 1 \leq\right.$ $i \leq d\}$ defined on $U$ such that, for every index $i$,

$$
\nabla \phi_{i} \equiv\left(h_{i 1}, \ldots, h_{i d}\right)^{T} .
$$

This follows easily from the assumption and the Poincaré Lemma [5, Theorem 8.1] (take $k=1$ and note every convex set is star shaped). It remains to show that there exists a function $\Phi$ such that

$$
\nabla \Phi=\left(\phi_{1}, \ldots, \phi_{d}\right)^{T}
$$

Note that for any pair of indices $i, j$, from equation (7) and the assumption, we have

$$
\frac{\partial \phi_{i}}{\partial x_{j}}=h_{i j}=h_{j i}=\frac{\partial \phi_{j}}{\partial x_{i}} .
$$

the existence of $\Phi$ is now established via the Poincaré Lemma.

Proof: (of Theorem 4) Step 1: Assume that $F_{x_{i} x_{j}}, F_{x_{i} y_{j}}$ and $F_{y_{i} y_{j}}, 1 \leq i, j \leq d$ are all continuous (i.e., $F$ is twice continuously differentiable). Fix arbitrarily $a, b \in \mathbb{R}^{d}$, and $p \in[0,1]$. Consider a random variable $X$ on some probability space $(\Omega, \mathcal{F}, P)$ such that $P(X=a)=p$ and $P(X=b)=q$ with $p+q=1$. Let $\mathcal{G}=\{\emptyset, \Omega\}$. Similar to the proof of equation (4), we have

$$
p F_{y_{i}}\left(a, y^{*}\right)+q F_{y_{i}}\left(b, y^{*}\right)=0, \quad \forall i=1, \ldots, d,
$$

at $y^{*}=p a+q b$. Taking derivatives over $p$ on both sides of the above equation and recalling $q=1-p$, we arrive at

$$
F_{y_{i}}\left(a, y^{*}\right)-F_{y_{i}}\left(b, y^{*}\right)+\sum_{j=1}^{d}\left[p F_{y_{i} y_{j}}\left(a, y^{*}\right)+q F_{y_{i} y_{j}}\left(b, y^{*}\right)\right]\left(a_{j}-b_{j}\right)=0
$$

for every $i=1, \ldots, d$. In particular, setting $p=1$ leads to

$$
F_{y_{i}}(a, a)-F_{y_{i}}(b, a)+\sum_{j=1}^{d} F_{y_{i} y_{j}}(a, a)\left(a_{j}-b_{j}\right)=0, \quad \forall i=1, \ldots, d .
$$

Since $F$ is non-negative and $F(x, x) \equiv 0$, we have $F_{y_{i}}(a, a) \equiv 0$. Write $H_{i j}(a) \doteq F_{y_{i} y_{j}}(a, a)$. Since $a$ and $b$ are arbitrary, the above equation is equivalent to

$$
F_{y_{i}}(x, y)=\sum_{j=1}^{d} H_{i j}(y)\left(y_{j}-x_{j}\right), \quad \forall x, y \in \mathbb{R}^{d} .
$$

Since $F_{y_{i}}$ is continuously differentiable for every $i$, it follows easily that $H_{i j}$ is also continuously differentiable for all $1 \leq i, j \leq d$. We now claim that there exists a function $\phi: y \in \mathbb{R}^{d} \mapsto H(y) \in \mathbb{R}$ such that

$$
\phi_{y_{i} y_{j}}(y)=H_{i j}(y), \quad 1 \leq i, j \leq d .
$$


Indeed, it follows from equation (8) that, for every $k=1, \ldots, d$,

$$
F_{y_{i} y_{k}}(x, y)=\sum_{j=1}^{d}\left(H_{i j}\right)_{y_{k}}(y)\left(y_{j}-x_{j}\right)+H_{i k}(y)
$$

and

$$
F_{y_{k} y_{i}}(x, y)=\sum_{j=1}^{d}\left(H_{k j}\right)_{y_{i}}(y)\left(y_{j}-x_{j}\right)+H_{k i}(y)
$$

Now, $F_{y_{i} y_{k}}=F_{y_{k} y_{i}}$ implies

$$
H_{i k} \equiv H_{k i}, \quad\left(H_{i j}\right)_{y_{k}} \equiv\left(H_{k j}\right)_{y_{i}} .
$$

The existence of $\phi$ now follows from Lemma 2 .

Moreover, equation (8) now becomes

$$
F_{y_{i}}(x, y)=\sum_{j=1}^{d} \phi_{y_{i} y_{j}}(y)\left(y_{j}-x_{j}\right)=\frac{\partial}{\partial y_{i}}[-\phi(y)-\langle\nabla \phi(y), x-y\rangle],
$$

which, combined with the condition $F(x, x) \equiv 0$, readily yields

$$
F(x, y)=\phi(x)-\phi(y)-\langle\nabla \phi(y), x-y\rangle=D_{\phi}(x, y)
$$

The convexity of $\phi$ follows from the non-negativity of $F$.

Step 2 and Step 3: Now repeating the same steps as those in the proof of Theorem 3 , the proof is complete.

\section{Conclusion}

Our paper provides necessary and sufficient conditions for loss functions under which the conditional expectation is the unique optimal predictor. Beyond its mathematical interest, the expansion form the $\mathbb{L}^{2}$-loss function to the general class of the BLFs has

its own distinctive value. In areas such as image and speech codings where the $\mathbb{L}^{2}$-loss function is no longer an appropriate or even meaningful measure of error (as was pointed out in [4]), other functions such as the well-known KL-divergence as well as the ItakuraSaito distance (Table 1)) play an dominant role. Our findings may serve as yet another mathematical justification for the adoption of these loss functions.

It is worth pointing out that through out the paper, for purpose of concise presentation, we assume that $X$ is allowed to take values in and the convex function $\phi$ is finte on, the whole Euclidean space $\mathbb{R}^{d}$. However, the same methodology with very minor modifications also works for the case when $\mathbb{R}^{d}$ is replaced by any open convex subset of $\mathbb{R}^{d}$. Some examples of interest include the half-space (for $\phi(x)=-\log x$ ), and the $d$-simplex (for $\left.\phi(p)=\sum_{j=1}^{d} p_{j} \log p_{j}\right)$. 
Finally, as was alluded earlier, the stronger regularity condition for the high-dimensional case (Theorem 4) is used in a crucial way to verify the compatibility condition (10) that seems almost necessary for solving the system of equations (9). It will be interesting to see if the regularity condition can be relaxed.

\section{References}

[1] K. B. Athreya. Prediction under convex loss. Technical Report 99-2, Department of Mathematics and Statistics, Iowa State University, 1999.

[2] A. Banerjee, S. Merugu, I. Dhillon, and J. Ghosh. Clustering with Bregman divergences. Technical Report TR-03-19, Department of Computer Science, University of Texas at Austin, 2003.

[3] L. M. Bregman. The relaxation method of finding the common point of convex sets and its application to the solution of problems in convex programming. USSR Computational Mathematics and Physics, 7:200-217, 1967.

[4] T. M. Cover and J. A. Thomas. Elements of Information Theory. Wiley-Interscience, 1991.

[5] C. H. Edwards. Advanced Calculus of Several Variables. Academic Press, 1973.

[6] I. Ekeland and R. Témam. Convex Analysis and Variational Problems. SIAM, Classics in Applied Mathematics, 1999.

[7] D. Gilbarg and N. Trudinger. Elliptic Partial Differential Equations of Second Order. Springer-Verlag, New York, 3nd edition, 2001.

[8] S. Karlin and H. M. Taylor. A First Course in Stochastic Processes. Academic Press, 2nd edition, 1974.

[9] E. L. Lehmann and G. Casella. Theory of Point Estimation. Springer, 2nd edition, 1998.

[10] R. T. Rockafeller. Convex Analysis. Princeton Landmarks in Mathematics. Princeton University Press, 1970.

[11] D. Williams. Probability with Martingales. Cambridge University Press, 1991. 